\title{
Development of Human Nervous Tissue upon Differentiation of Embryonic Stem Cells in Three-Dimensional Culture
}

\author{
Olivier Preynat-Seauve, ${ }^{a}$ David M. Suter, ${ }^{a}$ Diderik Tirefort, ${ }^{a}$ Laurent Turchi, ${ }^{b}$ Thierry Virolle,

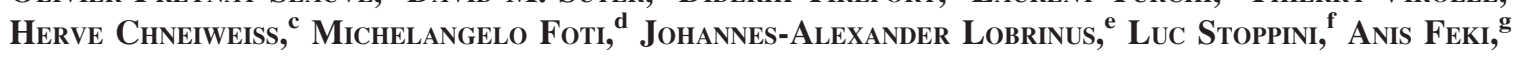 \\ Michel Dubois-Dauphin, ${ }^{a}$ Karl Heinz Krause ${ }^{a}$ \\ ${ }^{a}$ Department of Pathology and Immunology, Faculty of Medicine, University of Geneva and Department of \\ Genetic and Laboratory Medicine, Geneva Hospital, Switzerland; 'bINSERM U898, Faculty of Medicine, Nice, \\ France; ${ }^{\mathrm{c} I N S E R M ~ U 894, ~ F a c u l t y ~ o f ~ M e d i c i n e ~ P a r i s ~ D e s c a r t e s, ~ P a r i s, ~ F r a n c e ; ~}{ }^{\mathrm{d}}$ Department of Cell Physiology and \\ Metabolism, Faculty of Medicine, University of Geneva, Switzerland; ${ }^{\mathrm{e}}$ Neuropathology Unit, Department of \\ Genetic and Laboratory Medicine, Geneva Hospital, Switzerland; f Geneva Institute of Technology; HES-SO//

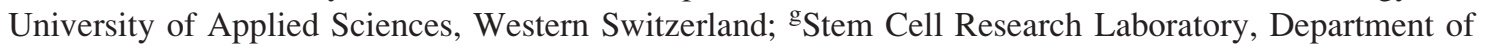 \\ Gynecology and Obstetrics, Geneva Hospital, Switzerland
}

Key Words. Developmental biology $\bullet$ Embryonic stem cell $\bullet$ Neural differentiation $•$ Nervous system

\begin{abstract}
Researches on neural differentiation using embryonic stem cells (ESC) require analysis of neurogenesis in conditions mimicking physiological cellular interactions as closely as possible. In this study, we report an air-liquid interfacebased culture of human ESC. This culture system allows three-dimensional cell expansion and neural differentiation in the absence of added growth factors. Over a 3-month period, a macroscopically visible, compact tissue developed. Histological coloration revealed a dense neural-like neural tissue including immature tubular structures. Electron microscopy, immunochemistry, and electrophysiological recordings demonstrated a dense network of neurons, astrocytes, and oligodendrocytes able to propagate signals. Within this tissue, tubular structures were niches of cells
\end{abstract}

resembling germinal layers of human fetal brain. Indeed, the tissue contained abundant proliferating cells expressing markers of neural progenitors. Finally, the capacity to generate neural tissues on air-liquid interface differed for different ESC lines, confirming variations of their neurogenic potential. In conclusion, this study demonstrates in vitro engineering of a human neural-like tissue with an organization that bears resemblance to early developing brain. As opposed to previously described methods, this differentiation (a) allows three-dimensional organization, (b) yields dense interconnected neural tissue with structurally and functionally distinct areas, and (c) is spontaneously guided by endogenous developmental cues. STEM Cells 2009; 27:509-520

Disclosure of potential conflicts of interest is found at the end of this article.

\section{INTRODUCTION}

Given the complexity of the central nervous system (CNS), the study of human brain development is a major challenge, which can only partially be addressed by extrapolation from animal experiments. Direct functional studies of human brain development are extremely difficult for ethical reasons. Thus, relevant in vitro models of human brain development are needed. The potential of human ESC to provide such models has been readily recognized; however, so far studies have focused on cellular development and not on tissue formation.
During embryogenesis, the CNS develops from neural progenitor cells (NPC) within the ectodermal germ layer. NPC are specified in space and time, becoming heterogeneous and generating a progressively restricted repertoire of mature neural cell subtypes [1]. The CNS originates as a sheet of cells made up of primary NPC, also referred as neuroepithelial cells. The latter forms the neural tube, easily discernable in humans by the end of the third week of gestation [2, 3]. The evolving neural tube is a germinative center containing NPC that self-renew and produce both neurons and glia. The neural tube germinative activity progressively decreases during development, the latter being replaced by the ventricular

Author contributions: O.P.-S.: conception and design, financial support, collection and/or assembly of data, data analysis and interpretation, manuscript writing, final approval of manuscript; D.M.S.: conception and design, financial support, data analysis and interpretation; D.T.: conception and design, data analysis and interpretation; L.T., T.V., and J.-A.L.: provision of study material, data analysis and interpretation; H.C. and A.F.: provision of study material; M.F.: provision of study material, data analysis and interpretation, financial support; L.S.: conception and design, data analysis and interpretation; M.D.-D. and K.-H.K.: conception and design, financial support, data analysis and interpretation, manuscript writing, final approval of manuscript.

Correspondence: Olivier Preynat-Seauve Ph.D., Departments of Pathology and Immunology, University of Geneva, Geneva, Switzerland. Telephone: +41 2237941 30; Fax: +41 2237941 32; e-mail: Olivier.Preynat-Seauve@medecine.unige.ch; or Karl Heinz Krause, M.D., Departments of Pathology and Immunology, University of Geneva, Geneva, Switzerland. Telephone: +41 223794130 ; Fax: +41 22 379 41 32; e-mail: Karl-Heinz.Krause@medecine.unige.ch Received July 3, 2008; accepted for publication November 26, 2008; first published online in Stem Cells Express December 11, 2008. (C) AlphaMed Press 1066-5099/2008/\$30.00/0 doi: 10.1634/stemcells.2008-0600

STem Cells 2009;27:509-520 www.StemCells.com 
system and spinal canal. Low numbers of NPC persist into the adult brain in the subventricular zone and the subgranular zone of the dentate gyrus in the hippocampus [4].

NPC in the evolving neural tube are radially oriented and contact both the apical (ventricular) and basal surfaces [1]. During brain development, they divide symmetrically or asymmetrically at the ventricular surface, forming a germinative center that produces radially neurons and glia [5]. By the onset of neurogenesis, neuroepithelial cells are progressively replaced by radial glial cells [6]. Radial glial cells divide in the evolving ventricular zone and produce neurons and glia $[7,8]$.

NPC can be derived in vitro from ESC and can be expanded in the presence of growth factors such as basic fibroblast growth factor (bFGF), epidermal growth factor (EGF), or leukemia inhibitory factor. Subsequently, they can be differentiated toward mature neurons, astrocytes, or oligodendrocytes through treatment with externally added factors [9-16]. A fraction of ESC-derived NPC spontaneously organize in vitro into rosettes; these neurogenic structures [10] are suggested to share some similarities with the neural tube. However, with the techniques applied so far, differentiation of NPC does not lead to a dense neuronal tissue; rather, the NPC grow as highly heterogeneous neural cell cultures.

This study demonstrates that in vitro expansion/differentiation of human ESC-derived NPC using an air-liquid interface system allows generation of an organized three-dimensional neural tissue. This tissue presents more phenotypic and structural similarities with the early developing human fetal brain than previously described methods.

\section{Materials ANd Methods}

\section{Culture of Undifferentiated ESC}

ESC cell lines $\mathrm{H} 1$ and $\mathrm{H} 9$ were from WiCell Research Institute (Madison, WI, http://www.wicell.org), and the HS-401 cell line was provided by Outi Hovatta, Karolinska Institute, Karolinska, Sweden). H1 and H9 were maintained in 80\% Dulbecco's modified Eagle's medium (DMEM)/Ham's F-12 medium (F12), 20\% Knockout serum replacement, $2 \mathrm{mM}$ L-glutamine, $1 \%$ nonessential amino acids, $0.1 \mathrm{mM} \beta$-mercaptoethanol, and $4 \mathrm{ng} / \mathrm{ml} \mathrm{bFGF}$. Mouse embryonic fibroblasts were used as feeders and isolated from embryos of pregnant CF-1 mice (Charles River Laboratories, Wilmington, MA, http://www.criver.com). Human foreskin fibroblasts were used as feeders for HS-401 and were from American Type Culture Collection (CCL-110; American Type Culture Collection, Manassas, VA, http://www.atcc.org). Fibroblasts were cultured in DMEM, $10 \%$ fetal bovine serum, and $1 \%$ penicillin/ streptomycin. Feeders were mitotically inactivated by irradiation (40 Gy) before being seeded on a gelatin-coated plate.

\section{Isolation of Gliomaspheres}

Viable fragments of high-grade human glioblastoma were transferred to a beaker containing $0.25 \%$ trypsin in $0.1 \mathrm{mM}$ EDTA (4:1) and slowly stirred at $37^{\circ} \mathrm{C}$ for $30-60$ minutes. Dissociated cells were plated in $75-\mathrm{cm}^{2}$ tissue culture flasks plated at 2,5005,000 cells per $\mathrm{cm}^{2}$ ) in DMEM/F-12 medium (1:1) containing the N2, G5, and B27 supplements (all from Invitrogen, Carlsbad, CA, http://www.invitrogen.com). After a delay ranging from 2 to 47 days, spheres bloomed from clusters of adherent cells and detached in the medium.

\section{ESC-Derived NPC Induction}

For the feeder-dependent method, MS5 stromal cell line (provided by Katsuhiko Itoh, Kyoto University, Kyoto, Japan) [17] were maintained in $\alpha$-minimal essential medium (Invitrogen) con- taining $10 \%$ fetal bovine serum and $2 \mathrm{mM}$ L-glutamine. NPC differentiation was induced by coculture with MS5. Five to 10 clusters were plated on a confluent layer of irradiated (50 Gy) MS5 in DMEM, 15\% Knockout serum replacement (Invitrogen), $2 \mathrm{mM}$ L-glutamine, $10 \mu \mathrm{M} \beta$-mercaptoethanol, and 1\% penicillin/streptomycin. After 2 weeks, cultures were switched for 2 additional weeks to N2 medium (DMEM with $4.5 \mathrm{~g} / \mathrm{l}$ glucose, N2 supplement [Gibco, Grand Island, NY, http://www.invitrogen.com], 10 ng/ml bFGF (Invitrogen), and 1\% penicillin/streptomycin).

ESC were also induced to NPC in feeder-independent conditions. Small ESC aggregates were induced in neural induction medium (DMEM/F12, N2 supplement [Gibco] and 1\% penicillin) during 4 days. Aggregates were then plated on $0.01 \%$ polyornithine, $1 \mu \mathrm{g} / \mathrm{ml}$ laminin-coated dishes containing neural induction medium.

\section{Air-Liquid Interface Expansion/Differentiation of NPC}

Rosette-enriched clusters were manually removed, and 5-10 clusters were plated on a hydrophilic polytetrafluoroethylene (PTFE) membrane (6 $\mathrm{mm}$ diameter, $0.4 \mu \mathrm{m}$; BioCell-Interface, La Chaux-de-Fonds, Switzerland, http://www.biocell-interface.com). This membrane was deposited on a Millicell-CM $(0.4 \mu \mathrm{m})$ culture plate insert (Millipore, Billerica, MA, http://www.millipore.com) (Fig. 1). One milliliter of N2 medium was added underneath the membrane insert.

\section{Neural Differentiation of ESC in Two Dimensions}

Generation of Neural Spheres. ESC were detached with type IV collagenase $(1 \mathrm{mg} / \mathrm{ml})$ and cultured in suspension in ultralowattachment plates (Corning Costar, Acton, MA, http://www. corning.com/lifesciences) for 3 weeks in neural induction medium.

Neural Differentiation in Adherent Conditions. Four-day neural spheres were plated at low density on $1 \mu \mathrm{g} / \mathrm{ml}$ laminincoated plates. After 1 week, rosette-enriched clusters were detached and dissociated with trypsin/EDTA before being replated at low density on laminin in neural differentiation medium (neurobasal supplemented with B-27 (all from Gibco) and $10 \mu \mathrm{g} / \mathrm{ml}$ human recombinant BDNF [R\&D Systems Inc., Minneapolis, http://www.rndsystems.com]).

\section{Immunofluorescence}

Samples were fixed in phosphate-buffered saline (PBS)/4\% paraformaldehyde for 30 minutes at room temperature, dehydrated, and embedded in paraffin. The sections $(10 \mu \mathrm{m})$ were then deparaffined, rehydrated, and heated in citrate buffer $(0.01 \mathrm{M}$; pH 6.0) in a $620-\mathrm{W}$ microwave oven for 15 minutes. The sections were then incubated overnight at $+4^{\circ} \mathrm{C}$ with appropriate dilutions of primary antibodies in PBS containing $0.2 \%$ Triton X-100 and $10 \%$ serum from the species corresponding to the secondary antibody. After washing in PBS, sections were incubated in PBS/ $0.2 \%$ Triton X-100 for 1 hour 30 minutes at room temperature with the appropriate dilution of secondary antibody, washed again, and incubated with $300 \mathrm{nM} \mathrm{4,6-diamidino-2-phenylindole}$ for 15 minutes. Sections were finally washed in PBS and rinsed with water before being mounted in FluorSave medium (Calbiochem, San Diego, http://www.emdbiosciences.com). For fetal brain analysis, the brains of human aborted fetuses were obtained from the Neuropathology Unit, in accordance with our institutional ethics comity.

\section{Real-Time Polymerase Chain Reaction}

Real-time polymerase chain reaction was performed on different old stage samples. Reactions were run on an ABI Prism 7900 HT detection system (Applied Biosystems, Foster City, CA, http:// www.appliedbiosystems.com). ALAS and GusB were used as housekeeping genes. As these genes behaved similarly in all samples examined, data were normalized to the $A L A S$ level. 

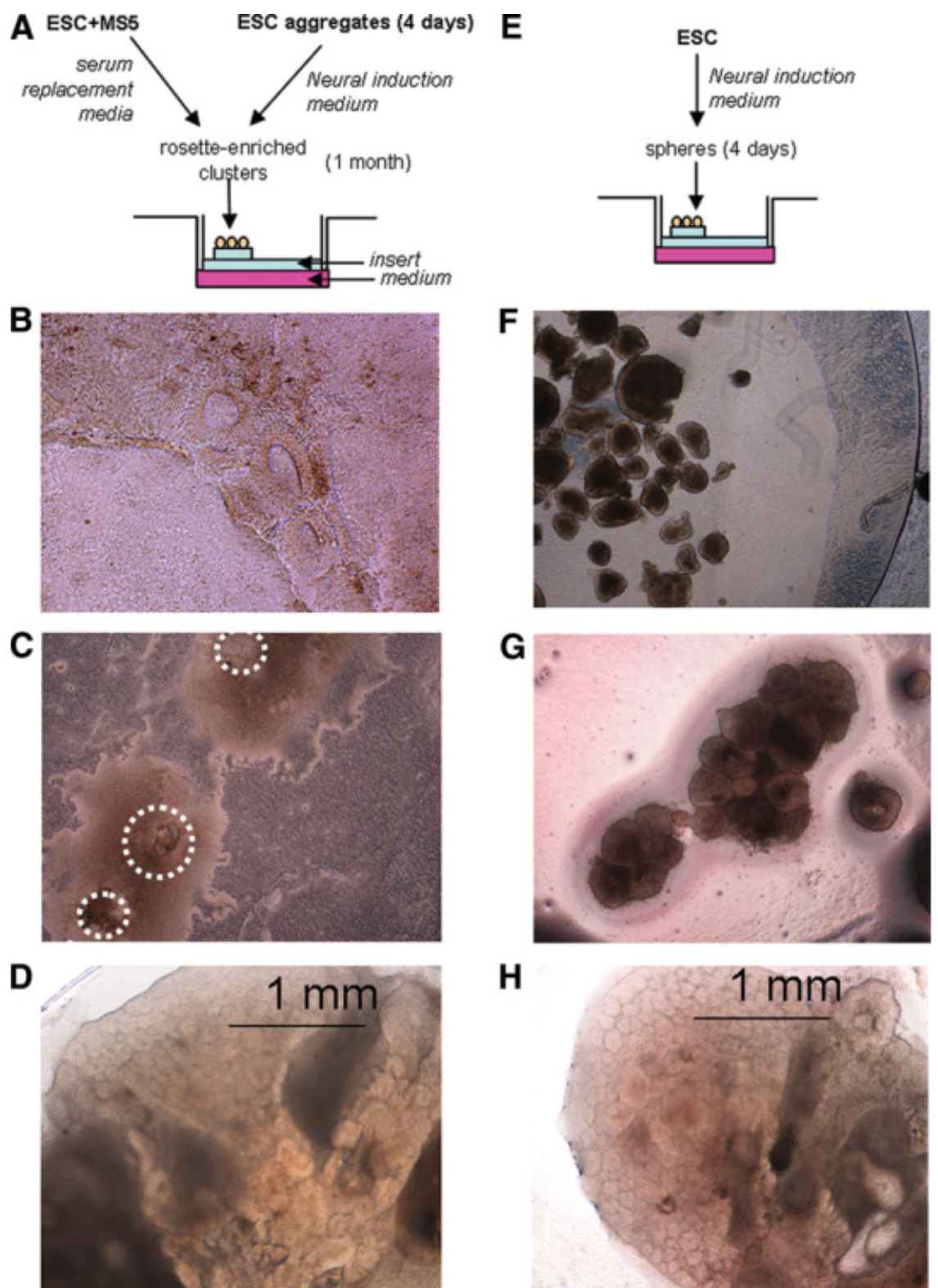

H
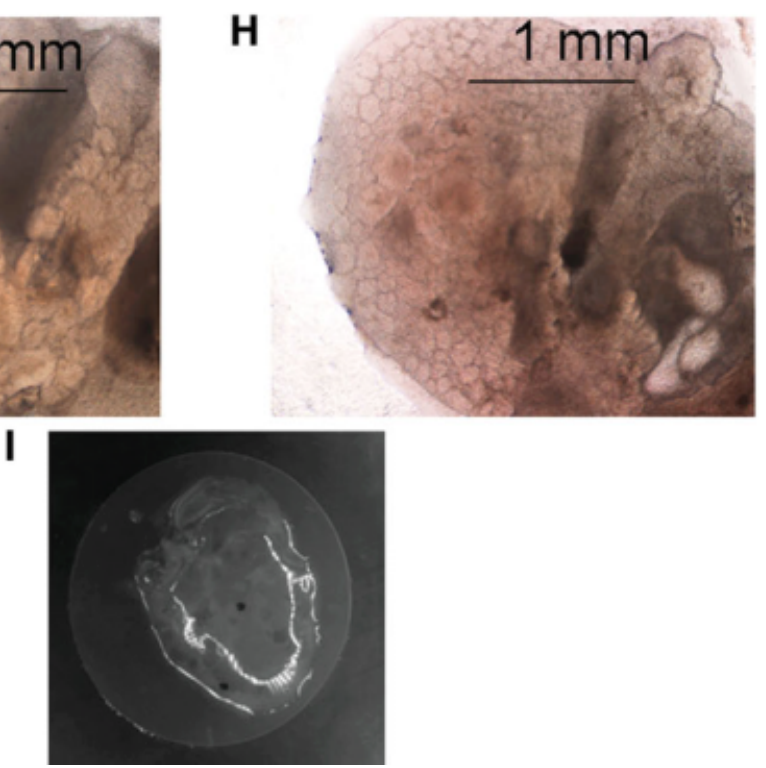

Figure 1. Three-dimensional air-liquid interface culture of ESC-derived neural precursors. (A): Schematic representations of air-liquid interface culture. Colonies of human ESC (H1 line) were induced to differentiate toward neural precursor cells through coculture with MS5 stromal cells or culture in chemically defined neural induction medium; after 1 month, rosetterich clusters were mechanically removed and plated on semipermeable membranes. (B-D): Expansion on membrane of MS5induced rosette clusters. Rosette cluster before removal and plating (B). (C): Cell growth 1 week after plating; dotted zones indicate the location of three initially plated clusters. (D): Development of a compact cell mass 1 month after plating, showing numerous newly formed rosettes. (E): Early aggregates of human ESC after 4 days of suspension culture in a chemically defined neural induction medium were directly plated on semipermeable membranes. (F-I): Expansion of early ESC aggregates. Ten to twenty 4-day-old ESC aggregates were plated $(\mathbf{F})$. Two days after plating, ESC aggregates attached and rapidly fused to form clusters $(\mathbf{G})$. One month after plating, ESC aggregates develop to a compact cell mass similar to that observed with rosette-rich clusters $(\mathbf{H})$. (I): Macroscopic view after 1 month of culture.

Sequences of the primers are described in supporting information Table 1.

\section{Electrophysiological Recordings}

Samples were transferred to a multi-electrode array system and maintained at $33^{\circ} \mathrm{C}$. The tissues were positioned so that their different regions were in contact with electrodes. Evoked field potentials could be recorded as described [18, 19].

\section{Transmission Electron Microscopy}

Fixation of the membrane-associated tissue was performed by incubation in 3\% glutaraldehyde for 2 hours. The fixed tissue was washed three times with PBS, dehydrated in ethanol, embedded in epoxy resin, and processed for electron microscopy as described previously [20]. Sections were contrasted with uranyl acetate and lead citrate and observed with a Technai 20 electron microscope (FEI, Eindhoven, The Netherlands, http://www.fei. com). 


\begin{abstract}
Antibodies
The following primary antibodies against human antigens were used: rabbit anti-cleaved caspase-3 (Cell Signaling Technology, Beverly, MA, http://www.cellsignal.com), mouse anti-cyclic nucleotide phosphodiesterase II (CNPase II), rabbit anti-Musashi, mouse anti-myelin oligodendrocyte-specific protein, rabbit antinestin, mouse anti-neuronal nuclei-specific protein $(\mathrm{NeuN})$, mouse anti-VGlut-1, mouse anti-vimentin, rabbit anti-Sox-1, mouse antisynaptophysin, rabbit anti-tyrosine hydroxylase (all from Chemicon, Temecula, CA, http://www.chemicon.com), goat anti-Pax-6, goat anti-doublecortin (all from Santa Cruz Biotechnology Inc., Santa Cruz, CA, http://www.scbt.com), mouse anti-proliferating cell nuclear antigen (anti-PCNA), rabbit anti-glial fibrillary acidic protein (anti-GFAP) (all from Dako, Glostrup, Denmark, http:// www.dako.com), mouse anti- $\beta$ III-tubulin (Sigma-Aldrich, St. Louis, http://www.sigmaaldrich.com), and rabbit anti- $\beta$ III-tubulin (Covance, Princeton, NJ, http://www.covance.com). The following fluorochrome-labeled secondary antibodies were used: Alexa Fluor (555 or 488)-labeled antibodies from goat or donkey against mouse, goat, or rabbit (Molecular Probes, Eugene, OR, http://probes.invitrogen.com).
\end{abstract}

\section{RESULTS}

\section{ESC-Derived NPC Expand on Air-Liquid Interface Cell Culture System}

ESC-derived NPC were induced by either feeder or feeder-independent conditions (Fig. 1A). The two methods allowed rosette cluster formation after 1 month (Fig. 1B). These rosettes consisted mainly of cells expressing NPC markers such as nestin, Sox-1, Musashi-1, and Pax-6 (data not shown). We proceeded them toward an air-liquid interface culture system of rosette-clusters similar to that previously used for tissue explants [21]. For that purpose, clusters were manually isolated and plated on precut patches of hydrophilic PTFE membranes before deposition on a culture insert (Fig. 1A). By addition of the culture medium only underneath the insert, the clusters were covered by capillarity by a very thin film of medium, allowing important air diffusion. One week after insert plating, neuroepithelial clusters had increased in size. Figure $1 \mathrm{C}$ shows a culture that consisted initially of four plated clusters that grew and merged within 1 week. After 1 month in culture, most of the initial cell clusters had merged, generating a compact cellular mass, covering the membrane, with numerous newly formed rosettes (Fig. 1D).

To investigate whether the method could be further simplified, we used early ESC aggregates obtained using a minimal neural induction medium (Fig. 1E). Such aggregates were only deposited on the membrane (Fig. 1F). After 2 days, they merged (Fig. 1G) and, after 1 month, developed into a cell mass (Fig. 1H, 1I).

\section{NPC Cultured on Air-Liquid Interface Develop into a Three-Dimensional Tissue}

Cell masses obtained by the three different methods were processed for histological investigation. In the case of feederinduced NPC, hemalun/eosin staining showed cells organized in a three-dimensional tissue-like structure (Fig. 2A). The tissue was heterogeneous, since immature mesenchymal zones (Fig. 2B) and dark pigmented zones (Fig. 2C) could be observed within the cell mass. Feeder-free induced rosettes (Fig. 2D) or directly plated ESC aggregates (Fig. 2E) generated tissues with significantly fewer mesenchymal and dark pigmented zones. Higher magnification revealed regions with distinct histological features. First, there were tubular struc- tures including radially oriented cells. Their dense nuclear staining and weaker cytoplasmic staining suggested immature structures (Fig. 2F, black arrow). Second, there were more mature zones devoid of organization, with less nuclear staining but more pronounced cytoplasmic staining (Fig. 2F, asterisk).

Other sources of NPC were also tested in their ability to generate a tissue using air-liquid interface cell culture system. Neurospheres including progenitors that were isolated from gliomas (H.C., manuscript in preparation) developed into a dense three-dimensional tissue (Fig. 2G). However, no organization was observed (Fig. 2H).

\section{Air-Liquid Interface Culture Induces a Neural Tissue Including Germinative Niches of NPC}

The phenotype of cells forming the tubes obtained in the liquid/air interphase culture was first analyzed in detail (Fig. 3).

- Many cells of the tube walls expressed PCNA, indicative of their mitotic activity. In contrast, mature zones contained few PCNA-positive cells (Fig. 3A).

- The radially organized cells forming the tubes were positive for molecules expressed in NPC: nestin, Musashi-1 (Fig. $3 \mathrm{~B}, 3 \mathrm{C})$. Note the radial organization of nestin-expressing filaments inside the tube walls (Fig. 3B). Pax-6 was the most documented transcription factor involved in neurogenesis and found to be expressed in the germinal layer of human developing fetal brain [22, 23]. Pax-6 was expressed in all cells forming tubes, confirming an NPC identity of such structures (Fig. 3D).

- Sox-1 was found in tubes and cells outside the tubes (few PCNA staining) (Fig. 3E). Cells within tubes coexpressed Pax-6and Sox-1 (Fig. 3F).

- Cells of the tube expressed also the intermediate filament vimentin but not the astrocytic protein GFAP (Fig. 3G).

- Brain lipid-binding protein (BLBP) was observed in proliferating cells, as well as filament around tubes (Fig. $3 \mathrm{H}$ ). In the developing mouse CNS, this protein has been correlated with neuronal differentiation in many parts of the mouse CNS, and studies have shown that BLBP is transiently expressed in radial glia in the embryonic ventricular zone (VZ) [24].

Together, these observations strongly suggest a proliferating NPC identity for cells within tubular structures. Such NPC resemble early rather than late fetal or adult brain NPC, inasmuch as they do not express astrocytic markers [1].

The nature of the tissue around tubes was next investigated. It was constituted of cells expressing the neuronal marker $\beta$ III-tubulin (Fig. 4A). The postmitotic cells being adjacent to tubes expressed the neuroblast marker doublecortin but not PCNA (Fig. 4B). The neuronal nature of such cells was confirmed by electron microscopy, showing densely packed neurites (Fig. 4C).

Strikingly, cells expressing the astrocytic markers GFAP were observed earliest after 2-month NPC culture (Fig. 4D). This delayed expression is reminiscent of the in vivo situation [1]. Moreover, the early oligodendrocyte marker CNPase II was expressed, suggesting the presence of immature oligodendrocytes within the differentiated tissue (Fig. 4E). In addition, coloration of tissue sections with the Luxol Blue and Bielchowski methods did not show any myelinization process (data not shown).

Some cells distant from tubes expressed NeuN (Fig. 4F) and synaptophysin (Fig. 4G), which are indicative of the mature stage of neuronal development. The glutamatergic and cholinergic nature of neurons was evidenced by 


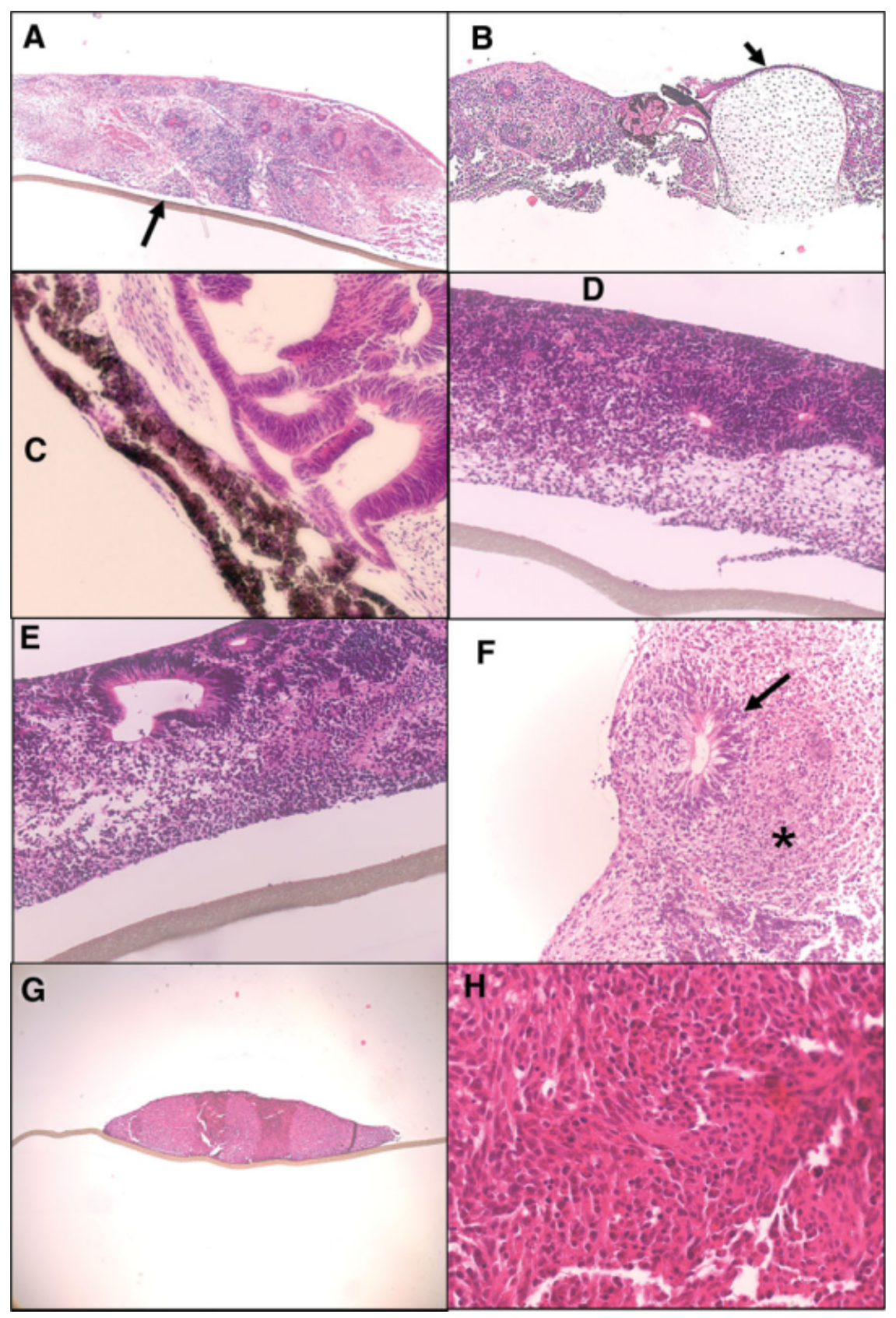

Figure 2. ESC or gliomasphere-derived neural progenitor cells develop into a compact tissue upon culture on air-liquid interface system. After 1 month of culture on a semipermeable membrane, cell masses were fixed, sectioned $(10 \mu \mathrm{m})$, and colored by hemalun/eosin. (A): Cell mass obtained from MS5-induced rosette-enriched clusters; the black arrow indicates the semipermeable membrane. (B): Immature mesenchyme-like structures (black arrow). (C): Dark pigmented zones. (D): Cell mass derived from rosette-enriched cultures obtained under chemically defined conditions. (E): Cell mass derived from ESC aggregates in chemically defined conditions. (F): The majority of the tissue consists of mature areas (strong cytoplasmic staining, asterisk) and immature radially organized structures (strong nuclear staining, black arrow). (G): Tissue section from the cell mass obtained after direct plating of gliomaspheres (1 month of culture). (H): Cells described in $(\mathbf{G})$ formed a compact tissue. immunostaining of the vesicular neurotransmitter transporters VGlut-1 (glutamate) and VAchT (acetylcholine) (Fig. 4H, 4I). Few tyrosine hydroxylase-positive neurons were observed (data not shown), indicating that dopaminergic and/or other catecholaminergic neurons were rare. The presence of mature neurons establishing connections was suggested by electron microscopy observation of structures resembling synapses (Fig. 4J) and the ability of the tissue to receive and propagate electrical signals. Indeed, successive electrical stimulations (2 $\mathrm{V}, 200$ microseconds) with 50 - or 10-millisecond intervals induced the propagation of an electrical signal that is typical of a group of neurons (Fig. 4K). It is noteworthy that the reduced propagation intensity after a short interval (10 milliseconds) indicated a paired-pulse inhibition process, suggesting the presence of a negative regulatory loop.
To confirm the neural phenotype of the ESC-derived tissue, expression of genes involved in early brain development was analyzed 2 and 4 weeks after plating on the insert. The pluripotency marker Oct-4 decreased upon air-liquid interface differentiation, whereas genes indicative of neural development, such as Pax-6, Pax-2, Pax-7, HoxB4, Nkx6.1, Olig-2, MAP-2, Sox-1, and Mash-1, increased (supporting information Fig. 1). Note the anterior, midbrain, and posterior identity of Pax-6, Pax-2, and HoxB4, respectively, and that Pax-7 is indicative of a dorsal identity. Mash-1 is a forebrain marker. The mesodermal marker Meox-1 increased upon differentiation probably accounted for by the observed regions of mesenchymal appearance. Olig2, which is indicative of oligodendrocyte lineage, increased also upon differentiation. However, differentiation toward radial glia-like progenitors was also 

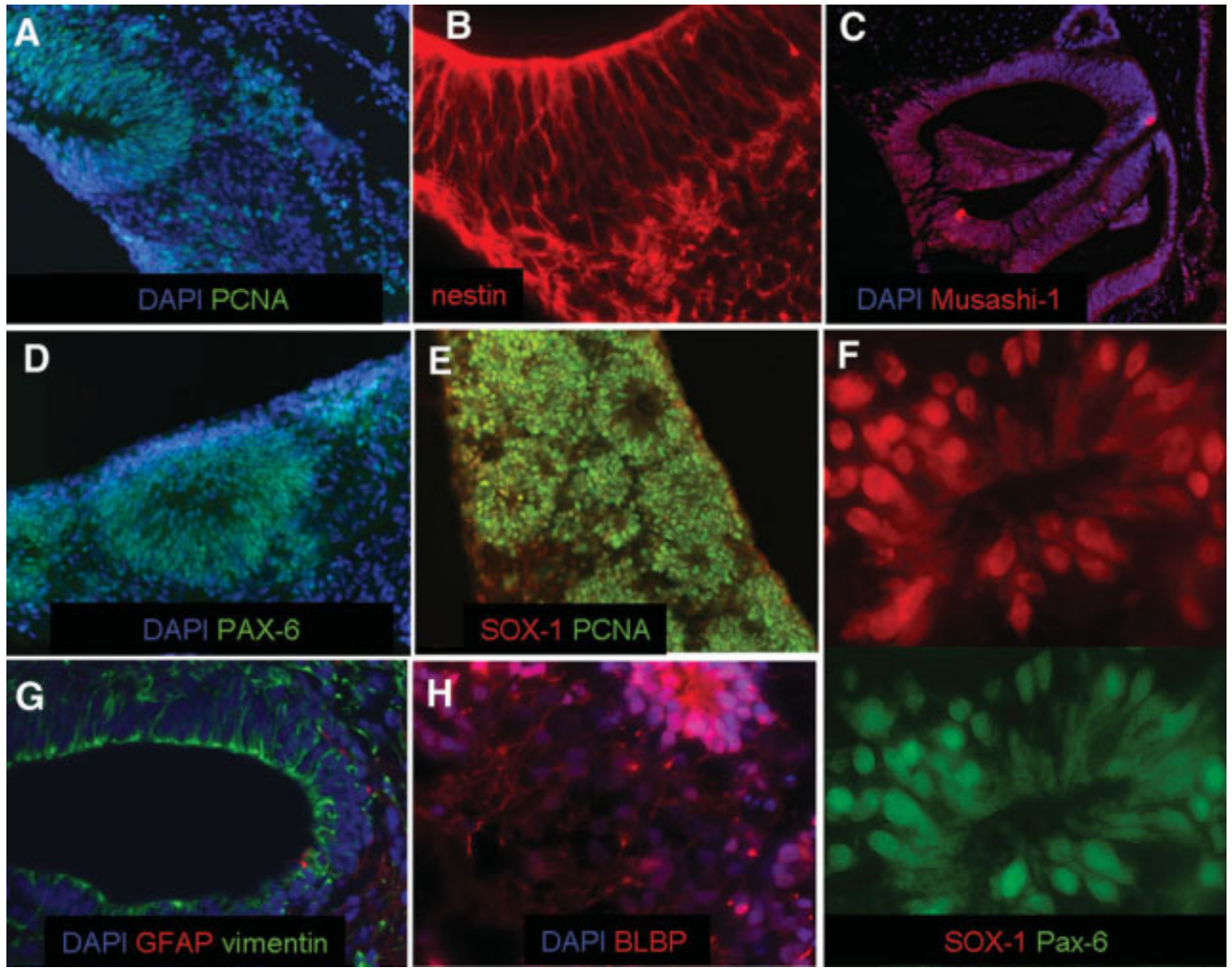

Figure 3. Tubular structures within engineered tissue harbor a neural progenitor cell phenotype. Tubular structures from a 2-month-cultured tissue section $(10 \mu \mathrm{m})$ were investigated for their phenotype. (A): Preferential expression of PCNA in tubes, rather than mature zones, confirms their germinal identity. (B): Nestin-expressing cells were radially organized in the tube wall. (C-E): Musashi-1 (C), PAX-6 (D), and Sox-1 (E) are expressed in cells of the tubes. (F): Cells of the tubes coexpressed Pax-6 and Sox-1. (G): Vimentin is radially arranged in cells of the tube. $(\mathbf{H})$ : BLBP is expressed in tubes and mature zones. Abbreviations: BLBP, brain lipid-binding protein; DAPI, 4,6-diamidino-2-phenylindole; GFAP, glial fibrillary acidic protein; PCNA, proliferating cell nuclear antigen.

suggested by this increase of Olig2 expression. Expression of the endodermal alpha foeto protein was found to be maintained. In contrast, the glial marker GFAP, the early mesodermal brachyury, the myelin-binding protein expressed by mature oligodendrocytes, and the endodermal Sox-17 were detected at very low level. Together, these observations confirm the neural identity but exclude organization according the positional identity along the dorsoventral or anteroposterior axis.

\section{Expression of the Used Markers in Human Fetal Brain}

With the ultimate goal of comparing the in vitro-induced tissue with the in vivo situation, the expression the neural markers described above was investigated in human fetal brain. Coronal sections at the level of the human periventricular germinal layer at different developmental stages (from 12 to 34 weeks; supporting information Fig. 2), including the adjacent cortical layers, were immunostained. VZ and subventricular zone (SVZ) were characterized by a high density of immature cells with a strong nuclear and a weak cytoplasmic staining (supporting information Fig. 2A-2D). Adjacent, there was the subplate (SP), including migrating young neuroblasts, with a lower density of nuclei and a more pronounced cytoplasmic staining (supporting information Fig. 2A, 2B). Neurons in the cortical plate (CP) could be localized at the 12- and 14-week stages (supporting information Fig. 2A, 2B). Germinal layers were clearly distinct from the other cortical layers at later stages (supporting information Fig. 2C, 2D). Results for all immunostainings at each stage are summarized in supporting information Table 1. As expected, cells of the germinal layer expressed the proliferation marker PCNA (e.g., 12 weeks; Fig. 5A) and the neuroepithelial marker nestin (e.g., 12 weeks; Fig. 5B). Note the radial organization of nestin expression close to the ventricle, similar to that observed in tubular structures. In fetal brain, the expression of Musashi-1 was strictly restricted to germinal layers (Fig. $5 \mathrm{C}$ ), as it was restricted to the germinal niches of ESCderived tissue. Sox-1, which was found in and outside germinal niches, was predominantly detected in nuclei of cells of the $\mathrm{CP}$, even though some cells of the SP were also positive (e.g., 12 weeks; Fig. 5D). Note that $\beta$ III-tubulin was outside germinative layers, being expressed as expected in SP and upper layers (e.g., 22 weeks; Fig. 5E). Such distinction between germinal and maturing layers was also observed in the induced tissue. Similarly, doublecortin immunoreactivity [25] was restricted to the SP (e.g., 12 weeks; Fig. 5F), being absent in germinal (Fig. 5F) and other layers (data not shown).

NPC are known to progressively generate astrocytes upon brain development, as shown by GFAP expression in late-stage fetal brain and adult SVZ [25-27]. In accordance with these previously described observations, expression of the GFAP was delayed. Indeed, GFAP was only weakly detected in germinative layers at week 14 (Fig. 6G), whereas late fetal stage (weeks 22, 34) showed a strong increase of the protein expression in the 

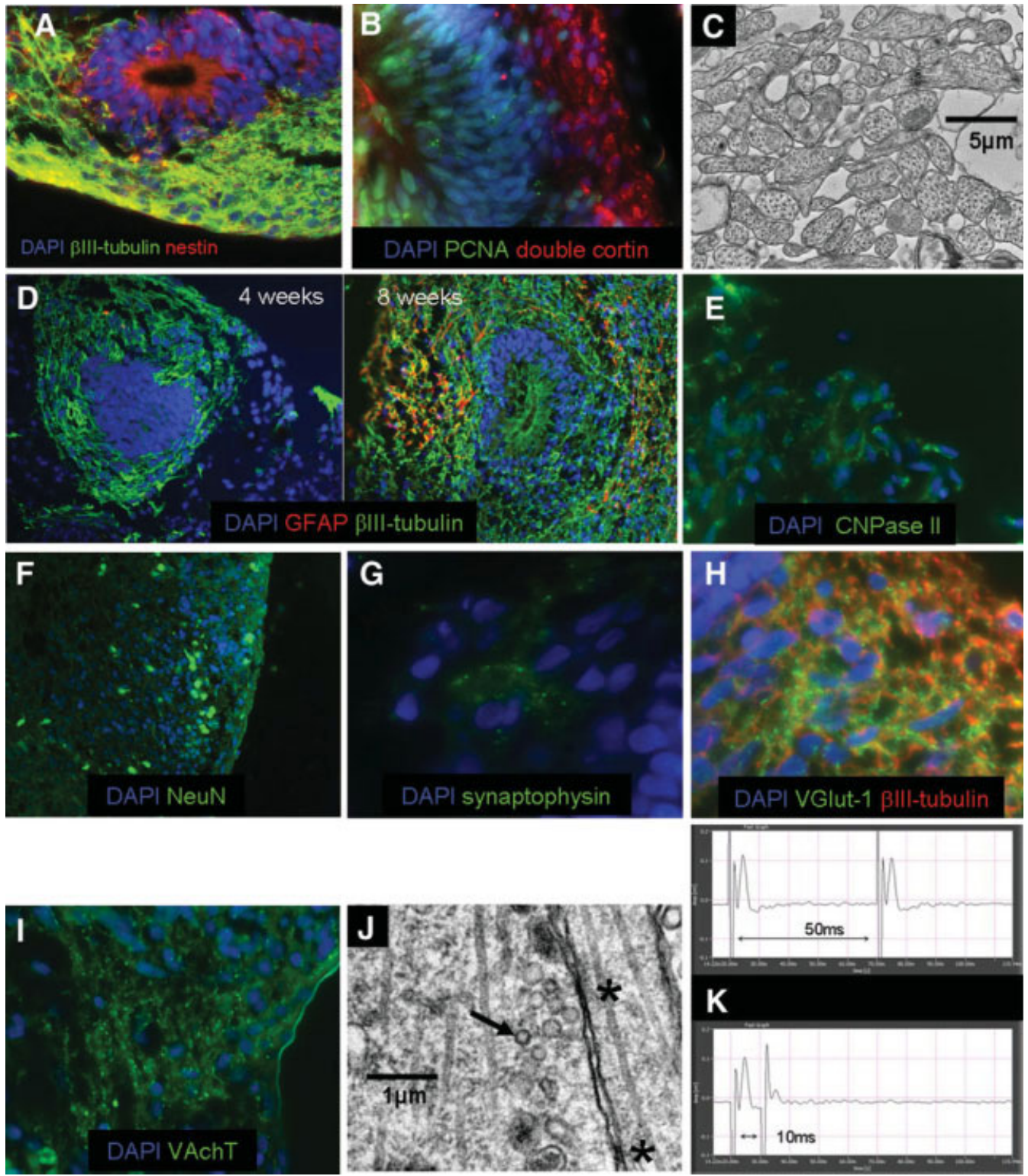

Figure 4. Characterization of extratubular regions in engineered tissue. (A): A neuronal network is found around tubular structures. (B): Neurons of the differentiated tissue around tubes are mainly young postmitotic neurons, since they express doublecortin and exclude PCNA staining. (C): Electron microscopy of ESC-induced tissue shows typical intermingled neurites. (D): Astrocytic cells were found, with a delayed appearance: glial fibrillary acidic protein-positive cells were not observed after 1 month of culture on the membrane (left) but were systematically observed within the neuronal compact tissue as of the 2nd month (right). (E): Young oligodendrocytes expressing CNPase II in cytoplasm are found within the mature tissue. (F): NeuN expression in cell bodies of the mature zones suggests the presence of late-stage neurons. (G): Synaptophysin expression in neuronal areas indicates the presence of mature neurons. $(\mathbf{H})$ : Most of neurons harbor a glutamatergic phenotype, as seen by the expression of VGlut-1. (I): VAchT staining indicates significant numbers of cholinergic synapses. (J): Electron microscopy of structures resembling synapses within the neuronal tissue. Vesicles in one side were observed (arrow), as well as increased density at the membrane level $\left.{ }^{*}\right)$. (K): Successive electrical stimulations (2 V, 200 microseconds) with 50- or 10-millisecond intervals induced the propagation of an electrical signal that is typical of a group of neurons. Abbreviations: CNPase II, cyclic nucleotide phosphodiesterase II; DAPI, 4,6-diamidino-2-phenylindole; NeuN, neuronal nuclei-specific protein; PCNA, proliferating cell nuclear antigen.

VZ (Fig. 5H). Similarly to GFAP, the expression of vimentin was delayed in the $\mathrm{VZ}$, being observed only at week 34 (Fig. 5I). In comparison, the absence of GFAP in germinal niches of the ESC-derived tissue suggests structures corresponding to early developmental stages, although the expression of vimentin does not correlate with the in vivo situation. Together, these observations reveal some phenotypic similarities between ESC-induced neural tissue and early fetal brain, especially at the inter- face between germinal and young migrating neuroblasts layers.

\section{Differences Between Differentiation on Air-Liquid Interface Culture and Two-Dimensional Methods in Cell Culture Dishes}

In vitro neural differentiation of ESC has been performed in numerous studies. We have compared in detail the air-liquid 

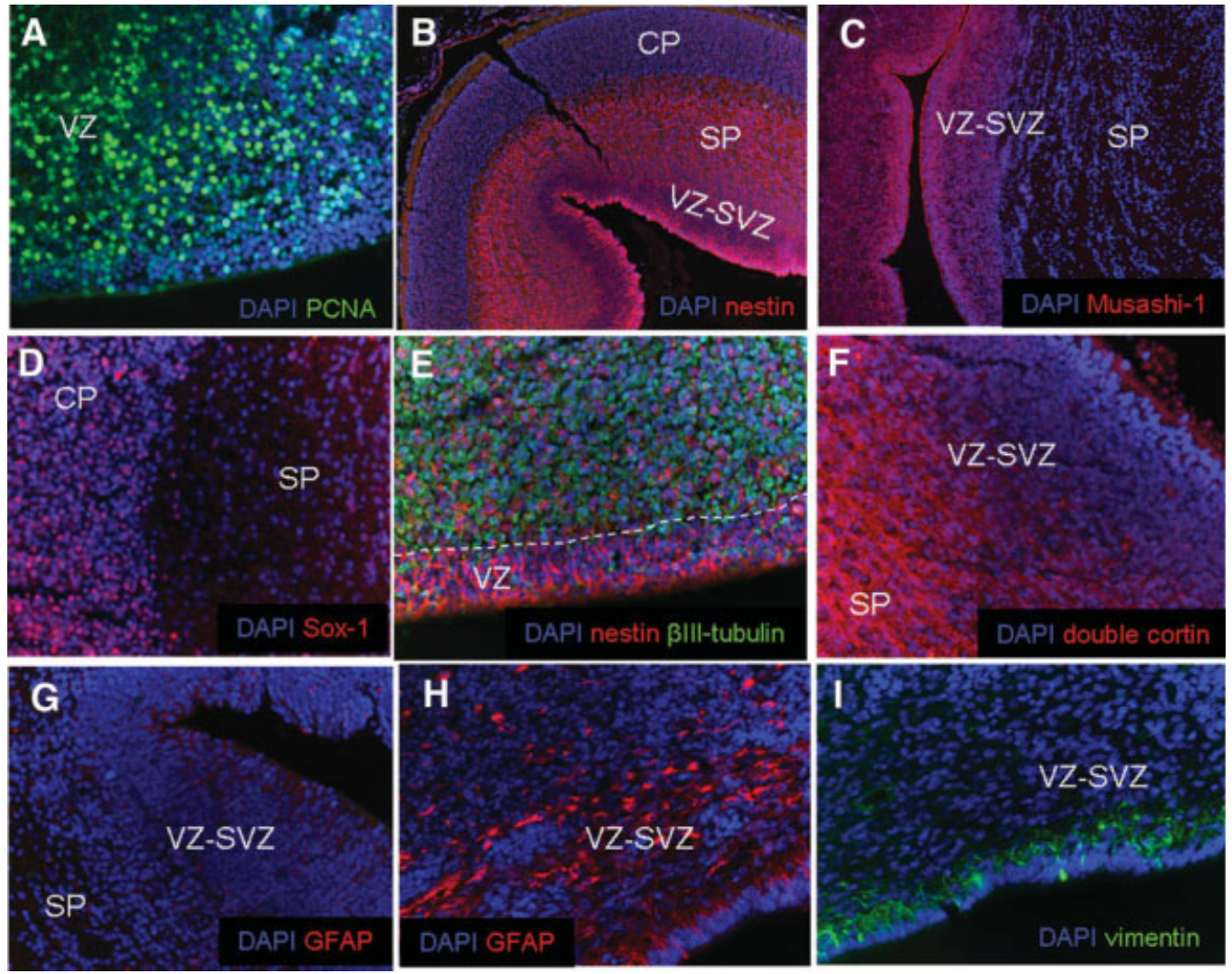

Figure 5. Structural organization and distribution of neural cell markers in germinal layers/mature tissue of human fetal brain. Sections (10 $\mu \mathrm{m}$ ) of human fetal brain at various developmental stages were analyzed by immunohistochemistry. Analysis was performed in fetal brain of 12 weeks (A, B, D, F, G), 14 weeks (C), 22 weeks (E, H, J), and 34 weeks (I). (A): Proliferating cell nuclear antigen-positive proliferating cells were found essentially in germinal layers. (B): Radially organized elongated cells in the VZ and cells within the SP expressed nestin. (C): Musashi-1 was expressed exclusively in VZ/SVZ. (D): Sox-1 was expressed mainly in cells of the CP, although it was also observed in some rare cells of the SP. (E): The neuronal marker $\beta$ III-tubulin is excluded from germinal layers but expressed in SP and other nonproliferating layers. (F): Doublecortin-expressing young migrating immature neurons are restricted to the SP. (G, H): Germinal layers of fetal brain at the 12-week-old stage (G) did not include GFAP-positive cells, whereas they were observed in radially organized cells of the VZ at week 34 (H). (I): The filament-associated protein vimentin was radially expressed in cells of the VZ. Abbreviations: CP, cortical plate; DAPI, 4,6-diamidino-2-phenylindole; GFAP, glial fibrillary acidic protein; SP, subplate; SVZ, subventricular zone; VZ, ventricular zone.

interface method with previously published studies. Globally, two kinds of methods were described: (a) neural differentiation of adherent cells, and (b) neural differentiation in suspension $[10,13,14,28-32]$. In the case of neural differentiation in adherent conditions, cells grew and differentiated as monoor paucilayers on feeder cells or substrate-coated plastic, inducing NPC that spontaneously organize into rosette and nonrosette structures. The cells included in rosettes are neuroepithelial NPC with a high neurogenic potential [10]. On the other hand, neural differentiation in suspension was shown to be efficient to obtain NPC and neurons inside three-dimensional clusters called "embryoid bodies," "spheres," or “aggregates" [9, 12, 13].

Expansion/differentiation of NPC on air-liquid interface shared some characteristics with previously published methods (self-organization of NPC into rosettes, a neuroepithelial phenotype, and mature neurons at the periphery of rosettes). However, the air-liquid interface-based method had unique properties that were not previously described.

(a) The number of rosettes for one air-liquid interface-derived tissue (supporting information Fig. 3A) was found to be significantly higher than that observed for one sphere in suspension (supporting information Fig. 3B). (b) Neural differentiation in suspension induced spheres that were highly heterogeneous in term of size and neurogenic potential. Indeed, some small spheres were found to resemble air-liquid interface-induced tissue: rosettes of neuroepithelial cells (nestin+) included in a dense tissue of mature neurons ( $\beta$ III-tubulin + ) (supporting information Fig. 3C). However, such structures were rare $(<10 \%)$ and mixed together with numerous other kinds of spheres, including non-neural tubular structures that were negative for nestin and few neurons (supporting information Fig. $3 \mathrm{D})$, as well as spheres without any neural features (nestin-, $\beta$ III-tubulin-) (supporting information Fig. 3E). Moreover, astrocytes and the expression of synaptophysin and doublecortin were not found within the small areas of neurons. In contrast, air-liquid interface culture was a more reproducible method, providing a tissue rich in NPC rosettes within a mature neural tissue.

(c) The heterogeneity of cell cultures is also a major feature of neural differentiation in adherent conditions. In addition to the absence of synchronization between cells starting/stopping differentiation, multiple types of structures resulted from spatial organization of cells. The following structures coexisted in culture during neuronal differentiation: rosette aggregates (supporting information Fig. 4A, black arrow), a monolayer of flat cells with an NPC 

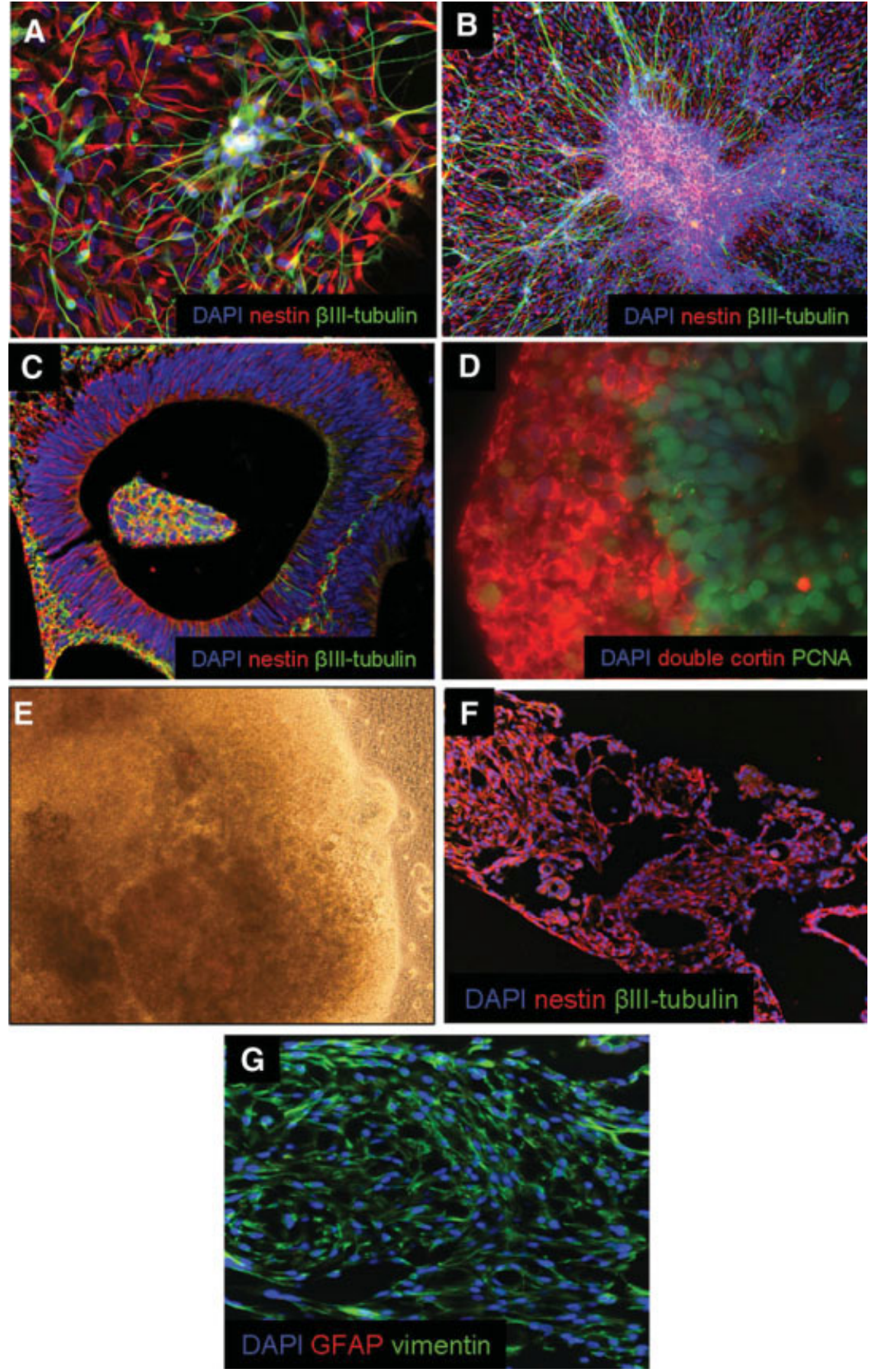

phenotype (supporting information Fig. 4A, white arrow), floating spheres that spontaneously reorganized (supporting information Fig. 4B), and neuronal scaffolds that were mixed with flat cells and aggregates (supporting information $4 \mathrm{C}$ ). Immunofluorescent staining confirmed that neurons $(\beta$ III-tubulin +$)$ were detected predominantly as dispersed cells that were intermingled with NPC (nestin+), apparently in a random fashion (Fig. 6A, 6B). In contrast, the air-liquid conditions favor compartmentalization between the two subpopulations (Fig. 6C), providing a more organized structure with a clear distinction between NPC niches and a dense neuronal network. Table 1 summarizes the differences between air-liquid inter-
Figure 6. Cell organization differs between the air-liquid interface and twodimensional method, as does the source of ESC/neural progenitor cells. (A, B): ESC differentiation in the two-dimensional cell culture system; monolayer of mature $\beta$ IIItubulin-positive neurons dispersed among nestin-expressing precursors (A); large cluster of nestin-expressing precursors surrounded by $\beta$ III-tubulin-positive neurons (B). (C): Distinct expression of nestin versus $\beta$ III-tubulin in air-liquid interface culture. (D): Immunofluorescent staining of PCNA and doublecortin of a section from a H9-derived tissue. (E): Cell mass obtained by culture of HS-401-derived sphere on a polytetrafluoroethylene membrane. (F, G): Immunostaining for nestin, $\beta$ III-tubulin (F), and vimentin (G) of HS-401-derived tissue. Abbreviations: DAPI, 4,6-diamidino-2-phenylindole; GFAP, glial fibrillary acidic protein; PCNA, proliferating cell nuclear antigen. face and both adherent and nonadherent conditions, previously described, for in vitro neural differentiation.

\section{Different ESC Lines Do Not Share the Same Capacity to Generate a Neural Tissue UnderAir-Liq- uid Interface Conditions}

The following ESC lines were compared for their ability to be differentiated using air-liquid interface system: H1, HS401, and H9. Each step of culture, as well as the phenotype of structures within the tissue, was compared, and observations are summarized in Table 1. H9-derived spheres plated 


\begin{tabular}{|c|c|c|c|c|c|c|}
\hline \multirow[b]{2}{*}{ Features } & \multicolumn{3}{|c|}{ Conventional method (H1 cell line) } & \multicolumn{3}{|c|}{ Air-liquid interface } \\
\hline & Adherent cultures & Spheres (1 week) & Spheres ( 2 weeks) & H1 & HS-401 & H9 \\
\hline Cell organization in three dimensions & $+1-$ & +++ & +++ & +++ & ++ & +++ \\
\hline Presence of rosettes & + & + & + & +++ & $+/-$ & +++ \\
\hline Heterogeneity & +++ & +++ & +++ & + & - & + \\
\hline Nestin & ++ & ++ & ++ & +++ & ++ & +++ \\
\hline Pill-tubulin & + & + & + & +++ & + & +++ \\
\hline \multicolumn{7}{|l|}{ Immunoreactivity } \\
\hline Pax-6 & ++ & ++ & ++ & ++ & - & ++ \\
\hline Sox-1 & ++ & ++ & ++ & ++ & - & ++ \\
\hline GFAP & + & - & - & $+1-$ & + & $+1-$ \\
\hline PCNA & + & + & + & + & + & + \\
\hline Doublecortin & $+1-$ & - & - & + & - & ++ \\
\hline Vimentin & + & + & + & + & + & ++ \\
\hline Synaptophysin & $+1-$ & & & +++ & - & +++ \\
\hline Dense neuronal tissue & - & - & + & +++ & - & +++ \\
\hline Astrocytes within neuronal tissue & - & - & - & + & - & + \\
\hline Segregation between niches and neuronal tissue & + & - & + & +++ & - & +++ \\
\hline Presence of non-neural cells & + & ++ & ++ & + & +++ & ++ \\
\hline
\end{tabular}

H1 ESC were either submitted to conventional neural differentiation in adherence or placed in suspension. Adherent neural cells were fixed and analyzed by immunofluorescence, whereas 1-week versus 3-weeks spheres obtained in suspension were embedded in paraffin before being immunostained. The immunoreactivity for several markers, as well as the features of neural differentiation, was compared. Several ESC lines were also compared under air-liquid interface conditions.

Abbreviations: GFAP, glial fibrillary acidic protein; PCNA, proliferating cell nuclear antigen.

on the membrane provided a tissue similar to that observed for $\mathrm{H} 1$ in term of cell organization and phenotype (Table 1). Figure 6D shows an example of a stained section from a H9derived tissue, where proliferating tubes $(\mathrm{PCNA}+)$ were distinct from a network of young migrating neuroblasts (doublecortin+). In contrast, HS-401 differed from $\mathrm{H} 1$ and $\mathrm{H} 9$. HS-401-derived spheres were able to grow and merge on the membrane, giving a tissue in three dimensions. However, the number of rosettes observed within the cell was dramatically low (Table 1). Cells within the tissue did not organize in tubes and were immunoreactive for nestin and vimentin (Table 1). No mature neurons expressing $\beta$ III-tubulin were observed. Thus, in line with studies reporting similar differences [33-35], this new system confirms the idea that ESC lines do not share identical capacity to generate neural cells. Finally, ESC-derived NPC were compared with those derived from adult glioma. Gliomaspheres differentiated on air-liquid interface culture induced a dense tissue including nestin and $\beta$ III-tubulin-positive cells (supporting information 5A). In this case, nestin-positive NPC and $\beta$ III-tubulin-positive neurons were mixed together without apparently organized structures. Note that the low organization of gliomasphere-derived tissue was confirmed using GFAP, vimentin, and PCNA staining (data not shown).

\section{Discussion}

\section{Air-Liquid Interface Culture and Differentiation Without Exogenous Factors}

Air-liquid interface culture was originally developed for organotypic culture of brain slices, and it has been shown to retain many essential organizational features of the host tissue [21]. Here we demonstrate that the technique can be adapted to perform three-dimensional expansion/differentiation of human ESC-derived NPC. An important feature of the air-liquid interface culture is the improved exchange between air and tissue, allowing the development of a relatively thick threedimensional culture without hypoxic cell death. In contrast with most of the previously described differentiation protocols [11, 13-16], the three-dimensional simplified method using ESC aggregates obtained in minimal conditions did not require exogenously added differentiating factors. Indeed, addition of fibroblast growth factor-2 or EGF did not induce phenotypic changes, although a moderate increase of the size of engineered tissue was observed. In addition, Noggin, a polypeptide playing a key role in neural induction, had no influence on the self-organization and phenotype of air-liquid interface-induced tissue. Thus, we suggest that this method at least favors spontaneous events driving the maintenance of NPC with mature neural cells.

The coexistence of proliferating stem cell niches and differentiated tissue is also remarkable in the sense that the proliferating areas do not invade the mature tissue (longest observation period, 4 months; data not shown). This suggests internal control, with stem cells remaining within tubes and radially migrating cells losing their capacity to proliferate.

\section{Expansion Versus Differentiation Using Air-Liquid Interface Conditions}

There is a difference between NPC expansion and neuronal/ glial differentiation. Previous reports demonstrated the expansion of NPC, as well as their differentiation into neurons and glia. However, although NPC, neurons, and glia always coexist in culture, there are different culture conditions available to favor expansion rather than differentiation (or the contrary). Air-liquid interface culture of NPC in a minimal medium favors neuronal/glial differentiation, associating in the same culture permanent niches of highly neurogenic NPC and highly mature neuronal tissue.

\section{The Role of Oxygen}

Oxygen changes appeared to have complex but poorly understood effects on precursor cell fate. Most in vitro studies on fetal precursors were performed in a nonphysiological oxygen tension (20\%). By comparison, lowered oxygen in the 
physiological range $(<5 \%)$ was shown to increase the expansion of NPC [36, 37]. Conversely, lowered oxygen was described to prevent neuronal differentiation of rat precursors [33]. Air-liquid interface is a noncomparable system, where a nanofilm of medium covers the tissue, favoring extensive gas exchanges between tissue and air. NeuN and especially synaptophysin were predominantly expressed in cells near the air side. It suggests that normoxia could favor the survival and/or differentiation of mature neuronal cells.

\section{Fetal Brain Versus ESC-Derived Brain-Like Tissue}

As shown in Results, there are similarities between the fetal brain and the tissues obtained in vitro. The in vitro-generated tissue reproduces the spatial dynamics of neurogenesis: radially organized, proliferating precursors in distinct germinal centers and production of differentiating neuroblasts, which migrate away from the luminal regions toward apical mature zones. As observed in fetal brain, the mature regions form a real parenchyma with abundant intermingled neurites. Expression of synaptophysin and markers of synaptic neurotransmitters demonstrates that the differentiated neurons express the molecular machinery that is required for synaptic transmission.

There are also interesting similarities between the tubular areas shown in the obtained tissue and the developing ventricular wall of the fetal brain. This is particularly true with respect to the radial organization of the nestin- and vimentincontaining filaments. The BLBP-, nestin-, and vimentin-containing cells resemble radial glia in some respects. The time frame of appearance of glial cells is another interesting feature. Indeed, in normal fetal brain, development of neurons precedes gliogenesis [1]. Similarly, the appearance of GFAPpositive astrocytes is observed earliest after 2 months of tissue formation. Immature oligodendrocyte were observed already after 1 month of tissue formation; however, myelinization was still absent after 2 months. Given the relatively short time of differentiation of the three-dimensional tissue, this is not surprising, as myelinization occurs only after 22 weeks in fetal brain (supporting information Table 1).

Among the differences between developing fetal brain and the three-dimensional culture, the presence of some apoptosis within the tubes was striking (data not shown). Such apoptotic cells were not observed in the fetal brain (data not shown). This apoptosis might be a peculiarity of our culture system, which limits the expansion of the germinative centers. However, alternative explanations should be considered: microglia/ macrophages, which are absent in the engineered tissue but present in the brain parenchyma as of the 4th month of development, might rapidly remove the zone of apoptotic cells in the fetal brain $[38,39]$.

Another element of fetal brain development that is not reproduced in our system is the establishment of the anterior to posterior and the dorsal to ventral axes. Although a set of positional markers was found, it is likely that the positional identity [40] is not respected by the process. As a logical con- sequence, there was also no spatial specification of defined neuronal subtypes groups.

\section{The Differences Among ESC Lines}

In line with other studies [34, 35, 41], this new system reinforces the idea that ESC lines do not share identical capacity to generate neural cells. These differences cannot be explained by chromosomal abnormalities since karyotypes were normal for the three tested lines. We consider the following explanations:

(a) Different epigenetic modifications, possibly due to different techniques of derivation and maintenance culture or due to heterogeneity of cells from the inner cell mass yielding embryonic stem cells with different properties;

(b) Genetic modifications (e.g., point mutations) that are not accompanied by karyotype changes;

(c) The genetic heterogeneity of the human population compared with the homogeneity of inbred mouse strains [34].

\section{Conclusion}

In conclusion, this study describes the engineering of threedimensional human nervous tissues from human embryonic stem cells. The system relies on spontaneous differentiation cues and appears to imitate, in space and time, some steps involved in early fetal brain development. It provides a novel and promising approach to study early events of human neurogenesis.

\section{ACKNOWLEDGMENTS}

We acknowledge Dr. K. Itoh (University of Kyoto, Japan), who provided the MS5 cell line. We thank the Electron Microscopy Core Facility at the Geneva Medical Faculty for access to electron microscope and ancillary equipments. This work was supported by the Clayton Foundation (to K.H.K.), Parkinson Schweiz (to O.P.-S. and M.D.-D.), the Swiss National Foundation (to D.M.S. and M.F.), and the Carlos and Elsie de Reuter Foundation (to M.F.).

\section{Disclosure of Potential Conflicts OF INTEREST}

L.S. owns stock in and served as an officer or member of the board for CAPSANT.

\section{REFERENCES}

1 Merkle FT, Alvarez-Buylla A. Neural stem cells in mammalian development. Curr Opin Cell Biol 2006;18:704-709.

2 Stern CD. Neural induction: Old problem, new findings, yet more questions. Development 2005;132:2007-2021.

3 Wilson SI, Edlund T. Neural induction: Toward a unifying mechanism. Nat Neurosci 2001;4(suppl):1161-1168.

4 Gould E. How widespread is adult neurogenesis in mammals? Nat Rev Neurosci 2007;8:481-488.

5 Haubensak W, Attardo A, Denk W et al. Neurons arise in the basal neuroepithelium of the early mammalian telencephalon: A major site of neurogenesis. Proc Natl Acad Sci U S A 2004;101:3196-3201.

6 Anthony TE, Klein C, Fishell G et al. Radial glia serve as neuronal progenitors in all regions of the central nervous system. Neuron 2004; 41:881-890.

7 Gotz M, Hartfuss E, Malatesta P. Radial glial cells as neuronal precursors: A new perspective on the correlation of morphology and lineage restriction in the developing cerebral cortex of mice. Brain Res Bull 2002;57:777-788

8 Malatesta P, Hack MA, Hartfuss E et al. Neuronal or glial progeny: Regional differences in radial glia fate. Neuron 2003;37:751-764. 
9 Cho MS, Lee YE, Kim JY et al. Highly efficient and large-scale generation of functional dopamine neurons from human embryonic stem cells. Proc Natl Acad Sci U S A 2008;105:3392-3397.

10 Elkabetz Y, Panagiotakos G, Al Shamy G et al. Human ES cellderived neural rosettes reveal a functionally distinct early neural stem cell stage. Genes Dev 2008;22:152-165.

11 Joannides AJ, Webber DJ, Raineteau O et al. Environmental signals regulate lineage choice and temporal maturation of neural stem cells from human embryonic stem cells. Brain 2007;130:1263-1275.

12 Joannides AJ, Fiore-Heriche C, Battersby AA et al. A scaleable and defined system for generating neural stem cells from human embryonic stem cells. Stem Cells 2007;25:731-737.

13 Nat R, Nilbratt M, Narkilahti S et al. Neurogenic neuroepithelial and radial glial cells generated from six human embryonic stem cell lines in serum-free suspension and adherent cultures. Glia 2007;55: 385-399.

14 Perrier AL, Tabar V, Barberi $\mathrm{T}$ et al. Derivation of midbrain dopamine neurons from human embryonic stem cells. Proc Natl Acad Sci U S A 2004;101:12543-12548.

15 Schulz TC, Noggle SA, Palmarini GM et al. Differentiation of human embryonic stem cells to dopaminergic neurons in serum-free suspension culture. Stem Cells 2004;22:1218-1238.

16 Yan Y, Yang D, Zarnowska ED et al. Directed differentiation of dopaminergic neuronal subtypes from human embryonic stem cells. Stem Cells 2005;23:781-790.

17 Itoh K, Tezuka H, Sakoda $\mathrm{H}$ et al. Reproducible establishment of hemopoietic supportive stromal cell lines from murine bone marrow. Exp Hematol 1989;17:145-153.

18 Thiebaud P, de Rooij NF, Koudelka-Hep M et al. Microelectrode arrays for electrophysiological monitoring of hippocampal organotypic slice cultures. IEEE Trans Biomed Eng 1997;44:1159-1163.

19 van Vliet E, Stoppini L, Balestrino $M$ et al. Electrophysiological recording of re-aggregating brain cell cultures on multi-electrode arrays to detect acute neurotoxic effects. Neurotoxicology 2007;28: 1136-1146.

20 Foti M, Mangasarian A, Piguet V et al. Nef-mediated clathrin-coated pit formation. J Cell Biol 1997;139:37-47.

21 Stoppini L, Buchs PA, Muller D. A simple method for organotypic cultures of nervous tissue. J Neurosci Methods 1991;37:173-182.

22 Bayatti N, Moss JA, Sun L et al. A molecular neuroanatomical study of the developing human neocortex from 8 to 17 postconceptional weeks revealing the early differentiation of the subplate and subventricular zone. Cereb Cortex 2008;18:1536-1548.

23 Mo Z, Zecevic N. Is Pax6 Critical for Neurogenesis in the Human Fetal Brain? Cereb Cortex 2008;18:1455-1465.

24 Feng L, Hatten ME, Heintz N. Brain lipid-binding protein (BLBP): A novel signaling system in the developing mammalian CNS. Neuron 1994;12:895-908.

25 Quinones-Hinojosa A, Sanai N, Soriano-Navarro M et al. Cellular composition and cytoarchitecture of the adult human subventricular zone: A niche of neural stem cells. J Comp Neurol 2006;494: 415-434.

26 Garcia AD, Doan NB, Imura T et al. GFAP-expressing progenitors are the principal source of constitutive neurogenesis in adult mouse forebrain. Nat Neurosci 2004; 7:1233-1241.

27 Doetsch F, Caille I, Lim DA et al. Subventricular zone astrocytes are neural stem cells in the adult mammalian brain. Cell 1999;97: 703-716.

28 Lee G, Kim H, Elkabetz Y et al. Isolation and directed differentiation of neural crest stem cells derived from human embryonic stem cells. Nat Biotechnol 2007;25:1468-1475.

29 Lee H, Shamy GA, Elkabetz Y et al. Directed differentiation and transplantation of human embryonic stem cell-derived motoneurons. Stem Cells 2007;25:1931-1939.

30 Sonntag KC, Pruszak J, Yoshizaki T et al. Enhanced yield of neuroepithelial precursors and midbrain-like dopaminergic neurons from human embryonic stem cells using the bone morphogenic protein antagonist noggin. Stem Cells 2007;25:411-418.

31 Vazin T, Chen J, Lee CT et al. Assessment of stromal-derived inducing activity in the generation of dopaminergic neurons from human embryonic stem cells. Stem Cells 2008;26:1517-1525.

32 Yang D, Zhang ZJ, Oldenburg $M$ et al. Human embryonic stem cellderived dopaminergic neurons reverse functional deficit in parkinsonian rats. Stem Cells 2008;26:55-63.

33 Gustafsson MV, Zheng X, Pereira T et al. Hypoxia requires notch signaling to maintain the undifferentiated cell state. Dev Cell 2005;9: $617-628$.

34 Osafune K, Caron L, Borowiak M et al. Marked differences in differentiation propensity among human embryonic stem cell lines. Nat Biotechnol 2008;26:313-315.

$35 \mathrm{Wu} \mathrm{H}$, Xu J, Pang ZP et al. Integrative genomic and functional analyses reveal neuronal subtype differentiation bias in human embryonic stem cell lines. Proc Natl Acad Sci U S A 2007;104:13821-13826.

36 Storch A, Paul G, Csete M et al. Long-term proliferation and dopaminergic differentiation of human mesencephalic neural precursor cells. Exp Neurol 2001;170:317-325.

37 Studer L, Csete M, Lee SH et al. Enhanced proliferation, survival, and dopaminergic differentiation of CNS precursors in lowered oxygen. J Neurosci 2000;20:7377-7383.

38 Rezaie P, Male D. Colonisation of the developing human brain and spinal cord by microglia: A review. Microsc Res Tech 1999;45: 359-382.

39 Mallat M, Marin-Teva JL, Cheret C. Phagocytosis in the developing CNS: More than clearing the corpses. Curr Opin Neurobiol 2005;15: 101-107.

40 Roussa E, Krieglstein K. Induction and specification of midbrain dopaminergic cells: Focus on SHH, FGF8, and TGF-beta. Cell Tissue Res 2004:318:23-33.

41 Allegrucci C, Young LE. Differences between human embryonic stem cell lines. Hum Reprod Update 2007;13:103-120.

See www.StemCells.com for supporting information available online. 